\title{
Intestinal Ischemia-Reperfusion: Rooting for the SOCS?
}

\author{
Younggeon Jin $^{1} \cdot$ Anthony T. Blikslager ${ }^{1}$
}

Published online: 9 November 2016

(c) Springer Science+Business Media New York 2016

Ischemia-reperfusion (IR) injury of the intestine occurs when blood flow is temporarily interrupted, which occurs during interventions such as surgery for an abdominal aortic aneurysm, small bowel transplantation, strangulating hernias, and neonatal necrotizing enterocolitis. It can also occur as a result of collapse of the systemic circulation during conditions such as hemorrhagic shock following trauma, septic shock, or heat stress [1]. At the cellular level, the initial intestinal ischemic injury reduces cellular mitochondrial ATP generation, activates hydrolases, reduces cell membrane selective permeability, and increases calcium influx into ischemic cells. Reperfusion may exacerbate the extent of injury through the activation of an intense systemic inflammatory response [2] such as marked pro-inflammatory cytokine release, production of reactive oxygen species (ROS), increased expression of nitric oxide (NO), Toll-like receptor (TLR)-4 signaling, and activation of inflammatory transcription factors, among other pro-inflammatory mechanisms [3]. IR injury is therefore difficult to manage clinically with consequent high morbidity and mortality [4].

Ischemic preconditioning is an alternative strategy to reduce IR injury among therapeutic interventions aimed at reducing IR injury by inhibiting the activation of inflammatory cells [4]. Ischemic preconditioning consists of a brief episode of ischemia preceding the major ischemic event, an intervention that activates tissue-adaptive mechanisms. Ischemic preconditioning can be induced mechanically or pharmacologically; mechanical

Anthony T. Blikslager

anthony_blikslager@ncsu.edu

1 Department of Clinical Sciences, College of Veterinary Medicine, North Carolina State University, 1060 William Moore Drive, Raleigh, NC 27607, USA preconditioning, in which the target organ is exposed to a brief ischemic episode by mechanically compromising its blood supply prior to prolonged ischemia, has the benefit of reducing IR injury, but it has the principal disadvantage of traumatizing major vessels and stressing the target organ. Alternatively, the identification of the signaling pathways underlying ischemic preconditioning has created the possibility of using pharmacological agents that confer protection against IR injury [5]. Ischemic preconditioning increases the generation of endogenous antioxidants such as glutathione, superoxide dismutase, and hemoxygenase-1 (HO-1). Nuclear transcription factors such as nuclear factor-kappa $\mathrm{B}(\mathrm{NF \kappa B})$ and $\mathrm{NO}$ are also affected by ischemic preconditioning, reducing the generation of pro-inflammatory cytokines, and preserving blood flow, oxygenation, and mitochondrial function [3, 6]. Clinically, ischemic preconditioning may be applied to patients prior to a planned surgical procedure such as cardiac, hepatic, or pulmonary surgery, in order to reduce the potential adverse effects of IR injury in the postoperative period.

In this issue of Digestive Diseases and Sciences, Liu et al. [7] report that ischemic preconditioning-induced suppressor of cytokine signaling-1 (SOCS-1) activation protects the intestine from IR injury via downregulation of the Toll-like receptor 4 (TLR4) pathway (Fig. 1). Among the molecular mechanisms integral to the pathogenesis of IR injury, the authors focused on TLR4 signaling and its downstream signaling intermediate tumor necrosis factor receptor-associated factor 6 (TRAF6), which has been understudied in IR injury. Additionally, the investigators also studied the contribution of receptor interacting protein 1 (RIP1), a key regulator of cellular apoptosis, regulated by tumor necrosis factor (TNF) signaling. SOCS-1 is a key regulator of inflammatory responses, including the TLR4 signaling pathway, thereby putatively inhibiting 


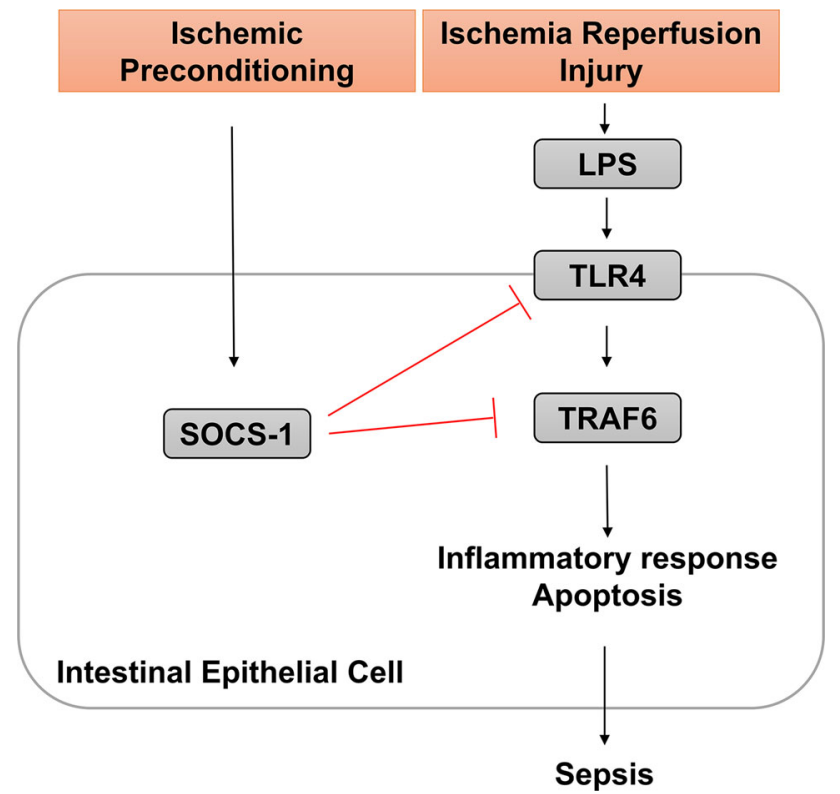

Fig. 1 Schematic of the signaling pathways studied by Liu et al., in which IR injury activates TLR-4 through exposure to luminal bacterial toxins such as lipopolysaccharide (LPS) in damaged intestine. TRAF6 activation results in downstream inflammatory responses typical of sepsis. In ischemic preconditioning, SOCS-1 is upregulated and thought to suppress TLR4 signaling, thereby having the potential to downregulate pro-inflammatory pathways in patients at risk of IR injury

inflammation and apoptosis. Thus, the authors hypothesized that SOCS-1 regulates TLR4-TRAF6 signaling, mechanisms important for ischemic preconditioning.

To investigate the contributions of TLR4-TRAF6 pathway and SOCS-1 to IR injury with or without ischemic preconditioning, the authors created an ischemic preconditioning and IR model system in rats by occluding the superior mesenteric artery. Just as the authors had postulated, intestinal injury was most severe with IR, whereas ischemic preconditioning substantially reduced the injury induced by IR. In terms of evaluating cell death during IR injury, the authors reported a significant increase in apoptotic cells after IR injury, substantially reduced in rats initially exposed to ischemic preconditioning. Additionally, the expression of TLR4 and TRAF6 increased gradually with increasing duration of intestinal ischemia, but increased strikingly after IR injury. Several recent studies have reported that the TLR4 pathway is closely associated with IR injury-induced epithelial cell apoptosis $[8,9]$. The authors addressed how TLR4 and TRAF6 act in concert to induce apoptosis and showed that IR increases apoptosis via TLR4 activation. The expression pattern of RIP1 was similar to that of TLR4 and TRAF6. Therefore, RIP1 likely is necessary for IR-induced inflammation and apoptosis in parallel with the TLR4-TRAF6 pathway. Importantly ischemic preconditioning inhibited these processes.
Perhaps of greatest interest, the expression of SOCS-1 gradually decreased during IR injury, but significantly increased after ischemic preconditioning, with the latter expression increase appearing to suppress epithelial cell apoptosis by inhibiting the TLR4 signaling pathway. Nevertheless, the relationship between SOCS-1 and the pro-inflammatory TLR4-TRAF6 signaling pathway is not entirely understood. To study this relationship in more detail, the authors reported that the mRNA expression of TRAF6 and SOCS-1 was inversely correlated during intestinal ischemia and IR, indicating that ischemic preconditioning may activate SOCS-1 and may suppress TLR4-TRAF6 signaling, protecting the intestinal mucosa from IR injury. From a translational point of view, these results indicate that SOCS-1 is a promising target for a potential pharmacological approach to ischemic preconditioning in patients at high risk of IR-related organ injury.

The study of these pathways from the perspective of local organ injury contributing to systemic inflammation is crucial since intestinal IR injury affects other vital organs such as lung, liver, and kidney through amplification of organ-specific inflammatory stimuli to systemic inflammatory responses. The expression of TLR4, TRAF6, RIP1, cleaved caspase- 3 and SOCS-1 in lung, liver and kidney tissue mirrored those of intestinal tissues after intestinal IR injury. These results indicate that the TLR4-TRAF6 pathway and the effects of SOCS-1 may participate in the regulation of multi-organ damage caused by intestinal IR injury.

This study provides essential information regarding the mechanisms of the regulation of inflammation and apoptosis during IR injury and ischemic preconditioning. Nonetheless, further studies are needed to fully understand the relationship between the TLR4-TRAF6 pathway and SOCS-1 on a more fundamental level. Of more significance to the medical community will be studies designed to address how SOCS-1 activation contributes to the protection associated with ischemic preconditioning in order to prevent IR injury and its complications.

\section{References}

1. Mallick IH, Yang W, Winslet MC, Seifalian AM. Ischemiareperfusion injury of the intestine and protective strategies against injury. Dig Dis Sci. 2004;49:1359-1377.

2. de Groot H, Rauen U. Ischemia-reperfusion injury: processes in pathogenetic networks: a review. Transpl Proc. 2007;39:481-484.

3. Camara-Lemarroy CR. Remote ischemic preconditioning as treatment for non-ischemic gastrointestinal disorders: beyond ischemia-reperfusion injury. World $J$ Gastroenterol. 2014;20: 3572-3581.

4. Vollmar B, Menger MD. Intestinal ischemia/reperfusion: microcirculatory pathology and functional consequences. Langenbeck Arch Surg Dtsch Ges Chir. 2011;396:13-29. 
5. Tapuria N, Kumar Y, Habib MM, Abu Amara M, Seifalian AM, Davidson BR. Remote ischemic preconditioning: a novel protective method from ischemia reperfusion injury-a review. J Surg Res. 2008;150:304-330.

6. Nandagopal K, Dawson TM, Dawson VL. Critical role for nitric oxide signaling in cardiac and neuronal ischemic preconditioning and tolerance. J Pharmacol Exp Ther. 2001;297:474-478.

7. Liu SZ, He XM, Zhang X, Zeng FC, Wang F, Zhou XY. Ischemic preconditioning-induced SOCS-1 protects rat intestinal ischemia reperfusion injury via degradation of TRAF6. Dig Dis Sci. (Epub ahead of print). doi:10.1007/s10620-016-4277-0.

8. Arumugam TV, Okun E, Tang SC, Thundyil J, Taylor SM, Woodruff TM. Toll-like receptors in ischemia-reperfusion injury. Shock. 2009;32:4-16.

9. Victoni T, Coelho FR, Soares AL, et al. Local and remote tissue injury upon intestinal ischemia and reperfusion depends on the TLR/MyD88 signaling pathway. Med Microbiol Immunol. 2010;199:35-42. 